\title{
Electronic structure of metallic and insulating phases of vanadium dioxide and its oxide alloys
}

\author{
Haichang Lu, ${ }^{1}$ Yuzheng Guo, ${ }^{2}$ and John Robertson ${ }^{1, *}$ \\ ${ }^{1}$ Department of Engineering, Cambridge University, Cambridge CB2 1PZ, United Kingdom \\ ${ }^{2}$ School of Engineering, Swansea University, Swansea, SA1 8EN, United Kingdom
}

(Received 6 June 2019; published 9 September 2019)

\begin{abstract}
$\mathrm{VO}_{2}$ attracts much attention due to its metal-insulator transition. Alloying $\mathrm{VO}_{2}$ with $\mathrm{MgO}$ and $\mathrm{GeO}_{2}$ allows the band gap and the transition temperature to be varied. We find that the spin order plays a key role in creating the band gap in the low-temperature $M_{1}$ phase. For $\mathrm{MgO}$ alloying, the alloying fraction $n\left(\mathrm{Mg}_{n} \mathrm{~V}_{1-n} \mathrm{O}_{2-n}\right)$ is varied from 12.5 to $33.3 \%$. The minimum band gap does not change without a structural rearrangement because both band edges of insulating $\mathrm{VO}_{2}$ consist of only $\mathrm{V} 3 d$ states on sixfold-coordinated $\mathrm{V}$ sites. A crystal search finds that if the $\mathrm{Mg}$ fraction in the alloy is large enough (>20\%), fivefold-coordinated V sites can have lower energy than the sixfold sites, and the band gaps are doubled. For $\mathrm{GeO}_{2}$ alloying, the insulating $M_{1}$ structure reverts to rutile because $\mathrm{GeO}_{2}$ has a rutile phase. The result matches the experimental observation and is very important in guiding $\mathrm{VO}_{2}$ 's applications such as smart coating and nonlinear resistor.
\end{abstract}

DOI: 10.1103/PhysRevMaterials.3.094603

\section{INTRODUCTION}

Pure vanadium dioxide $\left(\mathrm{VO}_{2}\right)$ has two phases; below $340 \mathrm{~K}$ it has the monoclinic or $M_{1}$ phase with a band gap, whereas above $340 \mathrm{~K}$ it has the metallic rutile form, called the $R$ phase [1-3]. The phase transition is fully reversible so that it is a promising material for various applications [4] such as smart windows [5], optical [6] or rf switches [7,8], and as a component of steep slope transistors [9-11]. Although the structural change at $340 \mathrm{~K}$ has been heavily studied, the electronic structures and especially the magnetic ground states of both $R$ and $M_{1}$ phases are still debated [1,3,12]. Experimentally, the magnetic order has been studied by NMR and electron paramagnetic resonance [12-15]. Theoretically, $\mathrm{VO}_{2}$ is described as a strongly correlated system [16,17], as a Mott insulator with some Peierls character [18], but the relative importance of these two factors is debated [19]. There are disputes to what extent density-functional theory (DFT) can describe the two phases [1,20]. Here, we find that the antiferromagnetic order with a strong spin coupling between chains of vanadium dimers plays a major role in generating the gap, favoring a Mott description [21].

Apart from understanding the physics of pure $\mathrm{VO}_{2}$, for applications it is of interest to vary the band gap of the insulating phase and vary the phase-transition temperature, while keeping the ability to switch between phases [22-24]. For instance, it is useful to shift the transition temperature $T_{\mathrm{C}}$ towards room temperature to enhance its suitability as a smart-window coating, or to raise the $T_{\mathrm{C}}$ to favor its use as a robust electrical or optical switch. It is also useful to be able to vary its optical gap for smart-window applications [5].

Experimentally, it is found that alloying $\mathrm{VO}_{2}$ with $\mathrm{MgO}$ will widen its $p$ - $d$ band gap to $2.3 \mathrm{eV}$ for alloying fraction $n=19 \%[22,23]$, whereas alloying $\mathrm{VO}_{2}$ with $\mathrm{GeO}_{2}$ will raise

\footnotetext{
*jr214@cam.ac.uk
}

its $T_{\mathrm{C}}$ [24]. However, so far there is little explanation of these effects in terms of electronic structure. Therefore, we carry out density-functional calculations of the electronic structure of pure and alloyed $\mathrm{VO}_{2}$ to provide this.

We separate the dopants into those like $\mathrm{Cr}$ or $\mathrm{Nb}$ which will dope $\mathrm{VO}_{2}$ in a substitutional fashion [2], where they change the $d$-electron count, to elements like Ge which behave like network-forming elements (in glass science) which alter the network bonding, to elements like $\mathrm{Mg}$ that behave like network modifiers which lower the coordination of the network.

It is interesting to compare alloying in $d$-electron systems like $\mathrm{VO}_{2}$ to $s, p$ bonded alloys like GaAs-AlAs whose band edges roughly interpolate between the energies of their end members. In $\mathrm{VO}_{2}$ alloys, the band-edge states are always formed from V $3 d$ states. For these, the number of occupied $\mathrm{V} 3 d$ states may change, but the band gap is controlled by the $\mathrm{V}-\mathrm{V}$ dimerization effect and spin coupling. As the band gap is determined mainly by the dimer itself, the magnitude of the band gap hardly changes, unless there is a change in the coordination of the V sites; in other words, it only varies by the action of the network-modifying elements.

\section{METHODS}

The calculation method is an important factor in the study of the alloys. The simple DFT method does not give a band gap for the $M_{1}$ phase [1]. In contrast, the more expensive methods such as GW or dynamic mean-field theory will reproduce the full electronic structure features [3] but are too computationally expensive for our case, given the need to use the large unit cells to treat dilute alloys.

Eyert $[1,20]$ noted that moderate cost methods such as hybrid functionals, which add a fraction of nonlocal (HartreeFock) exchange to the DFT local exchange-correlation function, can give a reasonable, bandlike description of the electronic states. Rubio and co-workers [25] noted that it is the inclusion of nonlocal exchange rather than strong correlations 
(a)

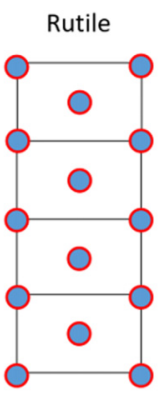

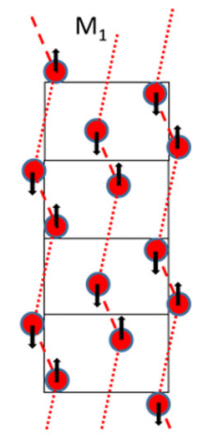

(b)

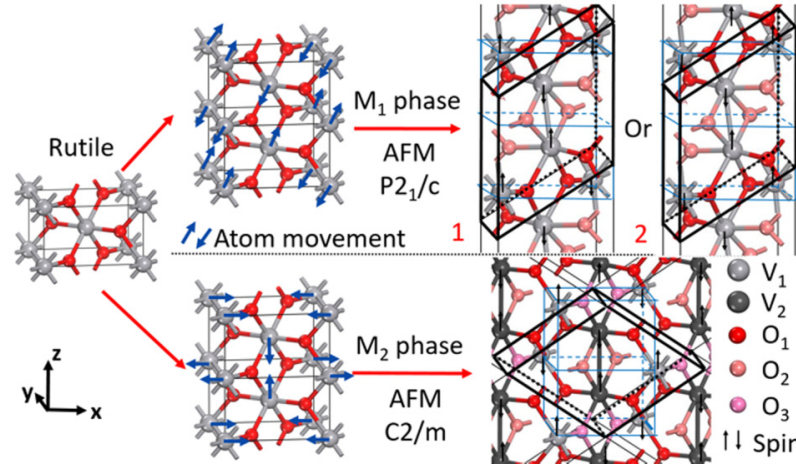

FIG. 1. (a) How the V sites spin pair as dimers and chains. (b) Structural transition from rutile phase to $M_{1}$ (up half) and $M_{2}$ (down half) phases via doubling the primitive rutile cell in $z$ axis. The blue arrows indicate how the $\mathrm{V}$ atoms shall move while the black arrow is the spin direction. The thin black lattice is the $1 \times 1 \times 2$ rutile lattice while the thick black lattice is the monoclinic lattice of $M_{1}$ and $M_{2}$.

which is the key factor to cure the local density approximation band-gap error for these oxides.

Eyert [20] used the Heyd-Scuseria-Ernzerhof hybrid functional [26] with the standard mixing fraction of $\alpha=0.25$. However, this gave a band gap larger than the experimental value of $0.6 \mathrm{eV}$ [21,27-29]. Schwingenschlögl and coworkers [30] noted that hybrid functionals with $\alpha=0.25$ give incorrect magnetic ground-state energies for both phases. Franchini [31] noted that hybrid functionals should use a lower value than $\alpha=0.25$ for such correlated oxide perovskites. Similarly, Pantelides and co-workers [32] noted that hybrid functionals with a lower fraction of $\alpha \sim 0.1$ would work for $\mathrm{VO}_{2}$. Recently, Zunger and co-workers [33] noted that allowing low-symmetry spin and structural states was a critical factor in the appearance of a band gap for related perovskite metal oxides, even when using relatively simple density-functional methods.

Our analysis finds the spin pairing in $\mathrm{V}-\mathrm{V}$ dimers is critical to opening up gaps. As regards whether $\mathrm{VO}_{2}$ is a MottHubbard or Peierls insulator, Huffman [21] noted that this corresponds in the Hubbard Hamiltonian to whether $U$ or the hopping term $t$ is dominant. It corresponds to whether the dimers are in the valence bond or molecular orbital limit. The role of fast lattice relaxations is seen in time-resolved studies $[34,35]$.

Our calculations were carried out using the CASTEP planewave density-functional code [36]. It has been noted that the GGA $+U$ method will give similar results to hybrid functionals for these open-shell metal oxides, so we use the GGA + $U$ method to study the atomic and electronic structure of the alloys. The Perdew-Burke-Ernzerhof form of generalized gradient approximation (GGA), including an onsite repulsive potential $U$ for $\mathrm{V} 3 d$ electrons was used. Values of $U$ from 2 to $3.25 \mathrm{eV}$ were tested and finally $U=2.5 \mathrm{eV}$ was used. Ultrasoft pseudopotentials with a plane-wave cutoff energy of $340 \mathrm{eV}$ are used. The geometry optimization algorithm is the two-point steepest descent for an initial rapid search of atomic structure, followed by the Broyden-Fletcher-Goldfarb-Shanno method for accurate structure calculation [36]. The relaxation is performed until the residue force is less than $0.03 \mathrm{eV} / \AA$. The density of $k$-point sampling varies for different cell size and is given in the Results and Discussion section. All calculations are spin polarized but spin-orbit coupling is excluded.

\section{RESULTS AND DISCUSSION}

\section{A. Pure $\mathrm{VO}_{2}$ : From rutile to $M_{1}$ and $M_{2}$ phase}

Firstly, we study how the insulating phases are derived from the rutile phase. We calculated structure, magnetic order, and partial density of states (PDOS) of each phase. There are two monoclinic phases $M_{1}$ and $M_{2}$.

The $M_{1}$ and $M_{2}$ phase together with the $T$ phase differ in the fraction of $\mathrm{V}$ sites that are dimerized, and how any dimers are arranged into chains, Fig. 1(a). In rutile $P 4_{2} / \mathrm{mnm}$, the metal-oxygen distances are equal. In $M_{1}$, all dimers form into a single type of chains along the $z$ axis of the rutile lattice. In $M_{2}$, half of the dimers make chains; half of the $\mathrm{V}$ sites are undimerized. The $T$ phase is intermediate between $M 1$ and $M 2$. Figure 1(b) shows how the monoclinic phases are formed from $1 \times 1 \times 2$ of primitive cells of the rutile phase.

TABLE I. Calculated lattice constant of various $\mathrm{VO}_{2}$ phase compared with previous work. Note that for $M_{2}$ the lattice constant of the conventional cell rather than the primitive cell is shown to compare to other papers.

\begin{tabular}{|c|c|c|c|c|c|c|}
\hline \multirow{2}{*}{$\begin{array}{l}\text { Lattice } \\
\text { constant }(\AA)\end{array}$} & \multicolumn{2}{|c|}{ Rutile } & \multicolumn{2}{|c|}{$M_{1}$} & \multicolumn{2}{|c|}{$M_{2}$} \\
\hline & This work & Ref. [1] & This work & Ref. [1] & This work & Ref. [1] \\
\hline$a$ & 4.62 & 4.55 & 5.67 & 5.75 & 9.05 & 9.06 \\
\hline$b$ & 4.62 & 4.55 & 4.61 & 4.54 & 6.03 & 5.80 \\
\hline$c$ & 2.78 & 2.85 & 5.44 & 5.38 & 4.52 & 4.52 \\
\hline$\alpha$ & 90.00 & 90.00 & 90.00 & 90.00 & 90.00 & 90.00 \\
\hline$\beta$ & 90.00 & 90.00 & 122.13 & 122.65 & 90.50 & 91.85 \\
\hline$\gamma$ & 90.00 & 90.00 & 90.00 & 90.00 & 90.00 & 90.00 \\
\hline
\end{tabular}


(a)

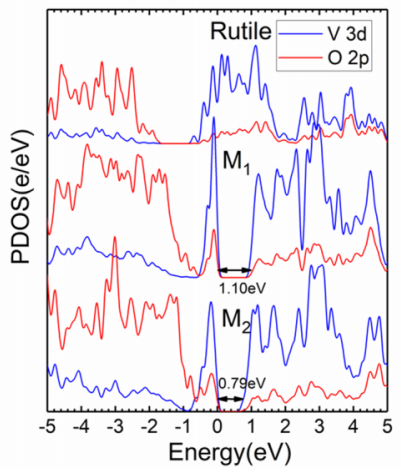

(b)
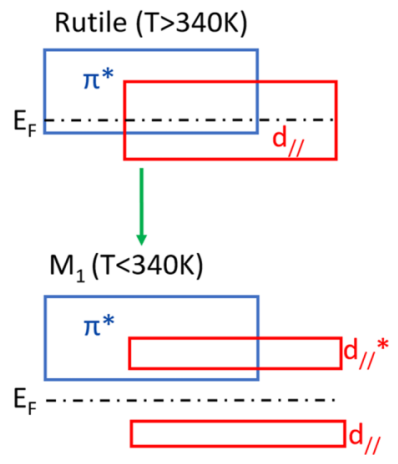

FIG. 2. (a) PDOS) of rutile, $M_{1}$, and $M_{2}$ phases. (b) Schematic band diagram of the metal-insulator transition [2].

The structures and cells of $R, M_{1}$, and $M_{2}$ are relaxed and the lattice constants of each phase are given in Table I with comparison to other papers. A $3 \times 3 \times 3 k$ point is used for the PDOS calculation shown in Fig. 2(a). The opening and closing of band gaps during metal-insulator transition are often attributed to a structural change. However, the gap is mainly induced by the spin pairing along the $\mathrm{V}$ chains which splits the $d_{/ /}$states as shown in Fig. 2(b). This follows because a gap exists in the rutile structure if it has antiferromagnetic (AFM) order, (unlike experimentally). On the other hand, a nonmagnetic monoclinic phase is gapless.

We find the ground state of monoclinic insulating phases should be AFM, where the chains of vanadium atoms have the opposite spin directions. In $M_{1}$, there are two spin configurations as in Fig. 1(b). Configuration 1 is that adjacent chains have the same spin orders while configuration 2 is that adjacent chains have opposite spin orders. While one can further make larger supercells and create more AFM configurations, this is unnecessary because the energy difference between configuration 1 and 2 is negligible and the difference of electronic structures are also negligible. In $M_{2}$, there is only one spin configuration shown in Fig. 1(a).

There is strong spin coupling between two chains of $\mathrm{V}$ atoms with opposite spin, while the two adjacent $\mathrm{V}$ atoms with the same spin direction are weakly coupled. We think the strength of spin coupling which is the distance between the $\mathrm{V}$ atoms with opposite spin is the key to determine the band gap. In $M_{1}$, all vanadium atoms lie in chains and strongly coupled with another $\mathrm{V}$ so a band gap of $1.10 \mathrm{eV}$ is calculated. In $M_{2}$, only $50 \%$ of $\mathrm{V}$ sites are in chains and strongly coupled; therefore, the band gap is calculated to be $0.79 \mathrm{eV}$. In rutile, no $\mathrm{V}$ atom is spin coupled; therefore, it is gapless. The PDOSs are shown in Fig. 2(a). Apart from the band gap, there should be a small gap in $M_{1}$ and $M_{2}$ phase which isolates the $d$ bands in the valence-band maximum (VBM) and $\mathrm{O} 2 p$ bands, as Eyert [1] argues.

For each $\mathrm{V}-\mathrm{V}$ chain, there is one $d$ band in the valence band (VB) known as $d_{/ /}$because the orbital is parallel to the chain direction, and nine $d$ bands in the conduction band (CB) which consist of both $d_{\| /}^{*}$ and $\pi^{*}$. These results are consistent with Goodenough's model [2]. If the temperature is close to $T_{\mathrm{C}}$, the energy difference between the $M_{1}$ and $M_{2}$ phase should be very small because the spin order is going to disappear. Apart from $M_{1}$ and $M_{2}$, the triclinic $T$ phase occurs as the intermediate phase between $M_{1}$ and $M_{2}$. The $T$ phase does not appear in our oxide alloys.

\section{B. MgO alloying of low-temperature monoclinic structures}

It is found experimentally that $\mathrm{MgO}$ doping can raise $\mathrm{VO}_{2}$ 's $p$ - $d$ band gap to at least $2.32 \mathrm{eV}$ as the ratio is $19 \%$ (a)

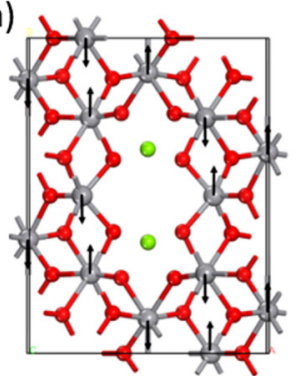

(c)

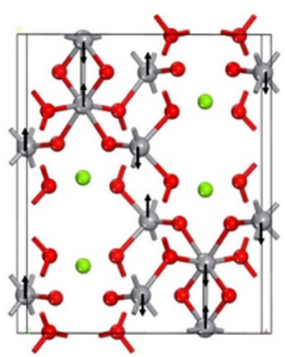

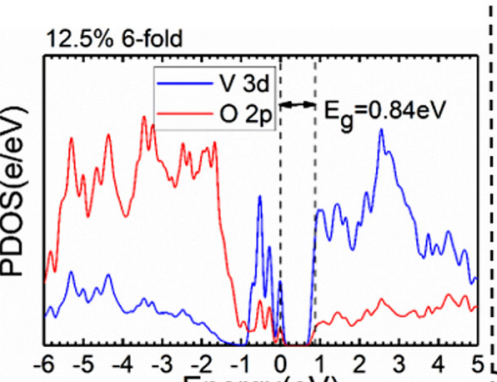
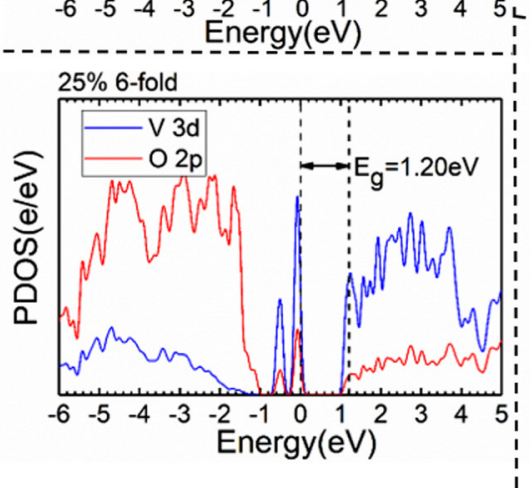

(b)
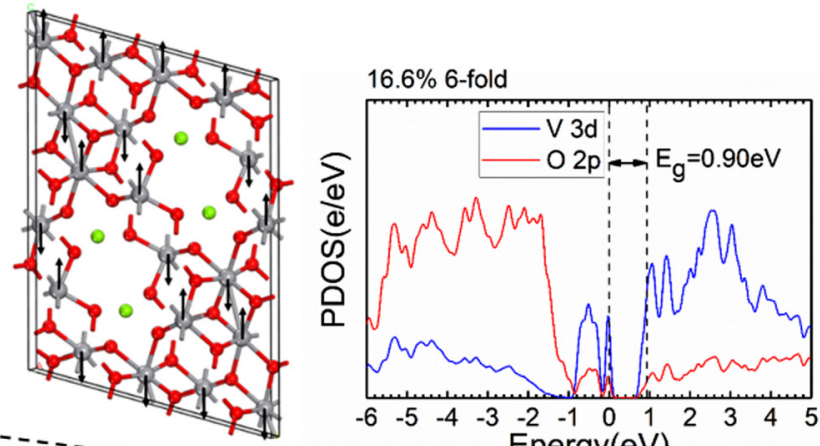

(d)

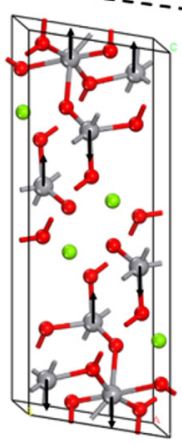
Energy $(\mathrm{eV})$

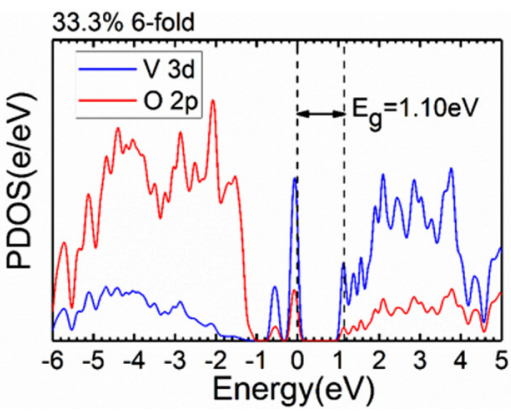

FIG. 3. Structure; spin configuration and PDOS of (a) $12.5 \%$, (b) $16.6 \%$, (c) $25 \%$, (d) $33.3 \% \mathrm{MgO}$ alloying $\mathrm{VO}_{2}$, sixfold. 
[22,23]. Other dopants like Al, Fe, Si have been tried, but there is no increase in the band gap [37]. Unlike them, $\mathrm{MgO}$ is highly ionic with a large band gap of $7.4 \mathrm{eV}$, and it has no state near Fermi level in the alloys. The lattice constant can be increased due to the larger spatial separation of $\mathrm{Mg}$ and $\mathrm{O}$. In this work, we choose alloying ratios of 12.5, 16.6, 25, and $33 \%$ for both $M_{1}$ and $M_{2}$ phase, as this creates supercell with a substitution ratios of 1:7, 1:5, 1:3, 1:2. One can either choose to substitute each $\mathrm{V}$ with $\mathrm{Mg}$ and delete one oxygen, or insert $\mathrm{MgO}$ into the supercell and do a geometry relaxation. These are the same and we choose the first method to help us obtain symmetric initial structures. We use a $1 \times 1 \times 3 k$-point mesh.

The relaxed structures of $\mathrm{MgO}$ alloys are shown in Figs. 3(a)-3(d). We find that irrespective of how $M_{1}$ or $M_{2}$ is alloyed, the final structures are the same and have nearly $50 \%$ of $\mathrm{V}$ dimer chains, which is $M_{2}$-like, maintaining sixfoldcoordinated V sites. Figure 3 shows the relaxed structures, spin order, and PDOS of the alloys with 12.5, 16.6, 25, and $33 \%$ of $\mathrm{MgO}$. We choose the initial centrosymmetric structures so that they can better comply with AFM. We do not break the $\mathrm{V}-\mathrm{V}$ chains as this would create $\mathrm{V}$ dangling bonds, so a 2-Mg unit is necessary. Furthermore, we distribute the 2-Mg units evenly in the supercell. We find all the alloys are AFM and with the $\mathrm{V}$ site sixfold, which means these structures form one of the energy minima. However, they are only one of the metastable states for $n>20 \%$. These structures are not necessarily the only alloying configurations for each ratio, but we find different configurations with centrosymmetry have similar electronic structures and their energy differences are negligible. We can see V $3 d$ bands in the VBM split due to the lowering of symmetry. The ratio of occupied and unoccupied $d$ bands is still 1:9. Figure 4(a) shows the trend of the ratio of V-V chains and the densities of alloys. Figure 4(b) shows the trend of the band gap. As $n$ increases, the V-V chain ratio decreases, the density decreases, and the band gap increases, by a small range. We find that unless the $\mathrm{V}-\mathrm{O}$ bonding changes, there is no significant change in the gap. This is inconsistent with experiment, so the sixfold structures energies are no longer the global minima. Figure 4(c) shows the difference in phase energy per $\mathrm{VO}_{2}$ formula unit.

As $n$ increases above $20 \%$, the structures undergo rearrangement. The $\mathrm{Mg}-\mathrm{O}$ bond is ionic and nondirectional, so the supercell can adopt the lowest energy. From crystal structure searches [38] we find that a lower energy with AFM exists. The energy differences per formula unit are shown in Fig. 4(c), stating that the new "fivefold structures" energies are $\sim 0.5 \mathrm{eV}$ per formula unit lower than their sixfold counterparts. Figures 5(a), 5(c), and 5(e) shows three fivefold cubic structures and spin orders with $n=20,25$, and $33.3 \%$. The symmetries increase from $P 2$ to $P 4 / n, P_{n m a}$, and $C_{m c m}$, respectively. The PDOS are also shown in Figs. 5(b), 5(d), and 5(f). The reconstruction helps $\mathrm{Mg}$ distribute more evenly among the supercell and $\mathrm{Mg}$ is no longer dimerized. The average spatial separation between $\mathrm{V}$ sites of opposite spin is smaller than for sixfold structures, so the spin coupling is increased. Figure 5(b) shows that the band gaps are nearly twice as large as the sixfold sites.

We also calculate the PDOS of the nonmagnetic fivefold structures in Figs. 4(b), 4(d), and 4(f), corresponding to hightemperature cases. We keep the structure while eliminating the
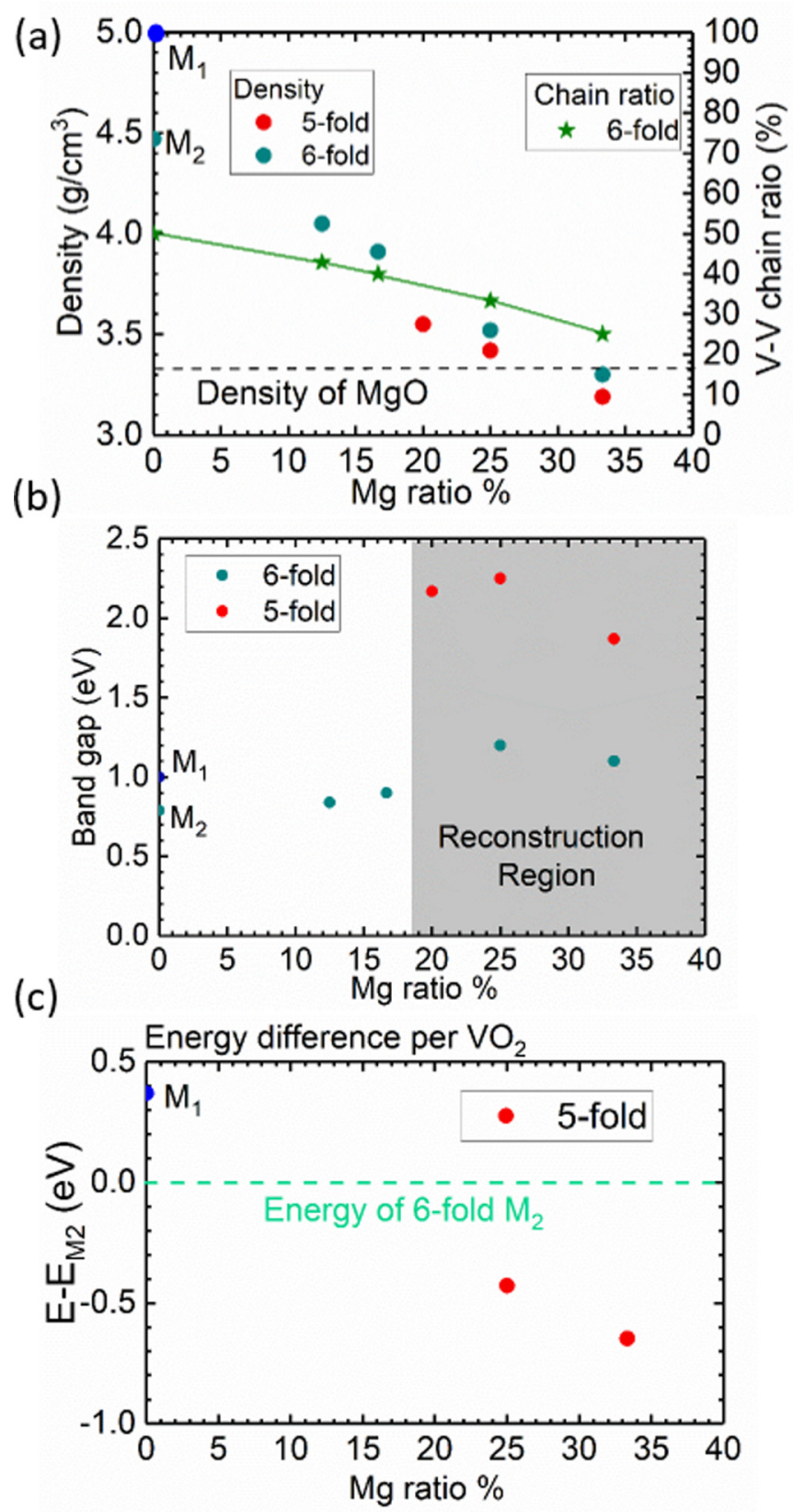

FIG. 4. (a) Density of alloys, $\mathrm{V}-\mathrm{V}$ chain ratio vs the $\mathrm{MgO}$ ratio. (b) The band gap vs the $\mathrm{MgO}$ ratio. (c) The energy difference of sixfold (M1) and fivefold $\mathrm{MgO}$ alloys per formula unit.

spin because the structural transition is negligible compared to magnetic order change. We find that as the spin disappears, the gap also disappears. Therefore, we note that the fivefold structures are potentially useful for phase-switch applications.

\section{C. $\mathrm{GeO}_{2}$ alloying}

We have also calculated the case of alloying $\mathrm{VO}_{2}$ with tetravalent Ge. A $25 \% \mathrm{GeO}_{2}$ alloy $\left(\mathrm{GeV}_{3} \mathrm{O}_{8}\right)$ is shown in Fig. 6. We initialize with AFM ordering and find that AFM is maintained during relaxation and a gap of $0.78 \mathrm{eV}$ is obtained. Unlike $\mathrm{MgO}$, the alloy structure reverts from $M_{1}$ to rutile structure, as shown in Figs. 6(c) and 6(d), where the 
(a)
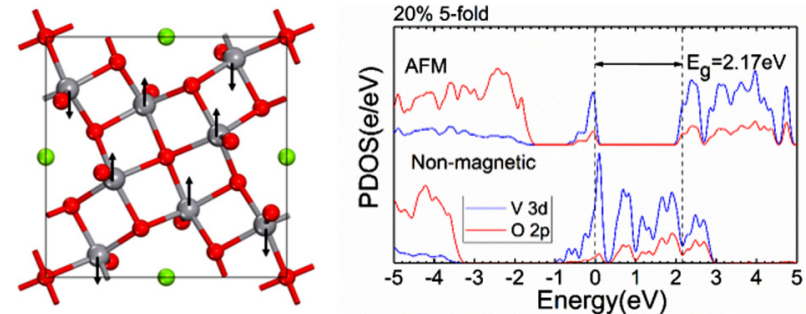

(b)

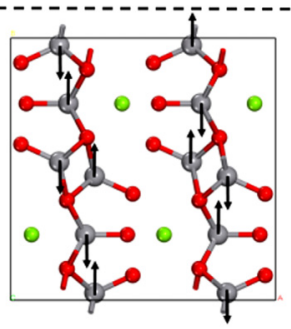

(c)
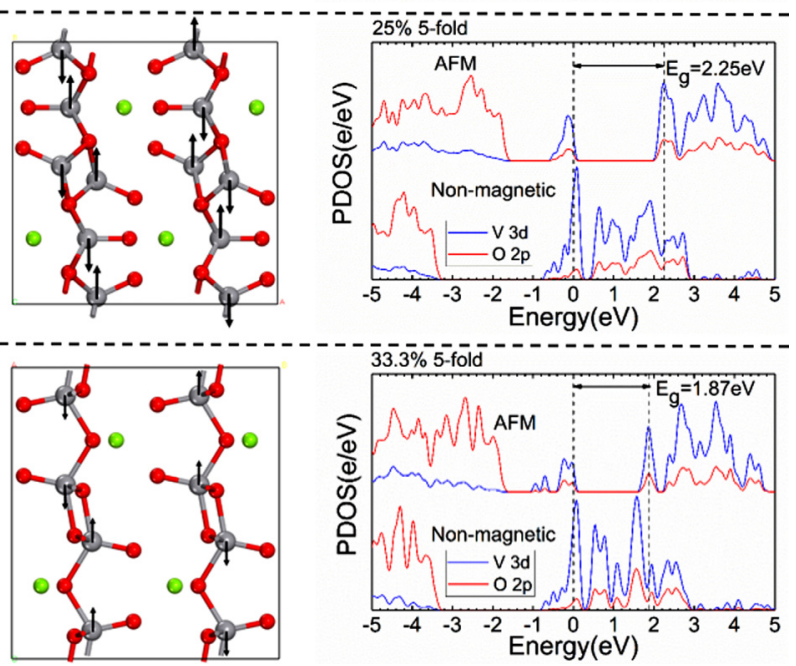

FIG. 5. Structure; spin configuration and PDOS of (a) 20\%, (b) $25 \%$, (c) $33 \% \mathrm{MgO}$ alloying $\mathrm{VO}_{2}$, with fivefold $\mathrm{V}$ sites.

side view shows that the $M_{1}$-type deformation disappears as $\mathrm{Ge}$ is doped. This is because $\mathrm{GeO}_{2}$ has a metastable rutile phase which allows the alloy to revert to rutile. However, the spin order, as shown previously, is relatively independent of structural order. If the temperature is low enough, the firstorder symmetry breaking will happen. Therefore, AFM is still (a)

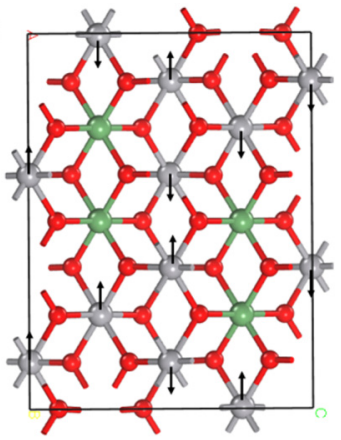

(c)

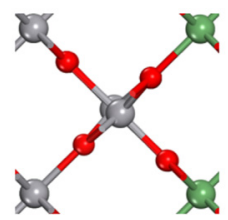

Before relaxation (b)

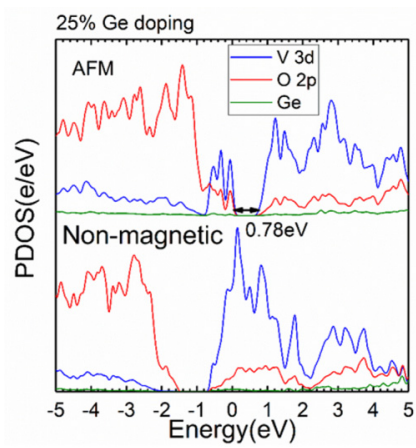

(d)

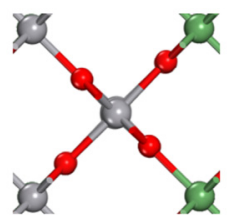

After relaxation
FIG. 6. (a) Structure, spin order and (b) PDOS of $25 \%$ $\mathrm{GeO}_{2}-\mathrm{VO}_{2}$ alloying. (c) Before and (d) after the geometric relaxation to show that the structure returns to rutile form. maintained in a low-temperature phase. We then predict that by controlling the amount of Ge doping, we can control the phase-change temperature. A calculation of the total energy with the nonmagnetic and AFM spin ordering finds that the energy difference between the metallic and nonmetallic phases increases, equivalent to a rise in transition temperature from 340 to $373 \mathrm{~K}$ for $25 \% \mathrm{Ge}$, as observed experimentally [24].

Figure 6(a) shows the spin ordering for the $\mathrm{VO}_{2}: \mathrm{GeO}_{2}$ alloy. Unlike the insulating phase of pure $\mathrm{VO}_{2}$, spin pairing along the $z$ axis occurs without any structural pairing of $\mathrm{V}-\mathrm{V}$ sites. The structure here assumes Ge atoms lie on one of the two chains. The spin pairing is weakened by the overall spatial separation of $\mathrm{V}$, thus reducing the gap to $0.78 \mathrm{eV}$. Krammer et al. [24] show that the transition temperature can be raised by Ge doping, indicating the magnetic ground-state energy is lower.

The transition temperature $T_{\mathrm{c}}$ depends linearly on the total energy difference per formula unit between the metallic and insulating phases. This increase in energy difference raises $T_{\mathrm{c}}$ for $\mathrm{GeO}_{2}$ alloying, which will be covered in detail elsewhere. An increase is consistent with experiment [24].

Generally, the spin alignment depends on the location of the dopant atoms within the chains of $\mathrm{V}$ sites, so that the $\mathrm{V}$ sites can pair up antiferromagnetically. However, the $\mathrm{V}$ pairing is able to occur for various spatial orderings of the dopant atoms. Thus $\mathrm{VO}_{2}$ (unlike $\mathrm{V}_{2} \mathrm{O}_{3}$ ) is not so sensitive to disorder, as noted both experimentally or theoretically $[18,39]$.

\section{CONCLUSION}

We have calculated the structure and electronic structure of pure $\mathrm{VO}_{2}$ and $\mathrm{MgO}, \mathrm{GeO}_{2}$ alloying $\mathrm{VO}_{2}$. We found that the spin order, which is AFM, is more important than structural change for the creation of the band gap. The spin coupling between chained V sites is crucial.

By alloying $\mathrm{MgO}$, we find a structural rearrangement happens as a high fraction of $\mathrm{MgO}$ is added, which causes the dramatic increase of the bonding and the band gap. This result is different from the previous view that $\mathrm{Mg}$ is a dopant. We emphasize that additives like $\mathrm{MgO}$ are unlike other dopants as $\mathrm{MgO}$ is highly ionic so it does not affect the electronic structure directly, but changes the bonding via encouraging structural reconstruction. Therefore, no midgap state is presented.

By substitutional doping with $\mathrm{Ge}$, which is same as $\mathrm{GeO}_{2}$ alloying, the insulating phase is returned to rutile, although the AFM and the gap are preserved because $\mathrm{GeO}_{2}$ is rutile.

This work provides a detailed structure and magnetic order study of $\mathrm{VO}_{2}$ and its metal-oxide alloying to explain the experimental observation.

\section{ACKNOWLEDGMENTS}

The authors acknowledge funding from Engineering and Physical Sciences Research Council (EPSRC) Grant No. EP/P005152/1 and European Commission, and discussions with S. J. Clark. 
[1] V. Eyert, Ann. Phys. (Leipzig) 11, 9 (2002).

[2] J. B. Goodenough, J. Solid State Chem. 3, 490 (1971).

[3] K. Liu, S. Lee, S. Yang, O. Delaire, and J. Wu, Mater. Today 21, 875 (2018).

[4] Z. Yang, C. Y. Ko, and S. Ramanathan, Ann. Rev. Mater. Res. 41, 337 (2011).

[5] S. Wang, M. Liu, L. Kong, Y. Long, X. Jiang, and A. Yu, Prog. Mater. Sci. 81, 1 (2016).

[6] P. Markov, R. E. Marvel, H. J. Conley, K. J. Miller, R. F. Haglund, Jr., and S. M. Weiss, ACS Photonics 2, 1175 (2015).

[7] W. A. Vitale, L. Petit, C. F. Moldovan, M. Fernandez, A. Paone, A. Schuler, and A. M. Ionescu, Sens. Actuators A 241, 245 (2016).

[8] E. A. Casu, N. Oliva, M. Cavalieri, A. A. Muller, A. Fumarola, W. A. Vitale, A. Krammer, A. Schuler, M. Fernandez, and A. M. Ionescu, IEEE J. EDS 6, 965 (2018).

[9] N. Shukla, A. V. Thathachary, A. Agrawal, H. Paik, A. Aziz, D. G. Schlom, S. K. Gupta, R. Engel-Herbert, and S. Datta, Nat. Commun. 6, 7812 (2015).

[10] T. Yajima, T. Nishimura, and A. Toriumi, Nat. Commun. 6, 10104 (2015).

[11] W. A. Vitale, E. A. Casu, A. Biswas, T. Rosca, C. Alper, A. Krammer, G. V. Luong, O. T. Zhao, S. Mantl, A. Schuler, and A. M. Ionescu, Sci. Rep. 7, 355 (2017).

[12] A. X. Gray et al., Phys. Rev. Lett. 116, 116403 (2016).

[13] J. P. Pouget, H. Launois, T. M. Rice, P. Dernier, A. Gossard, G. Villeneuve, and P. Hagenmuller, Phys. Rev. B 10, 1801 (1974).

[14] J. P. d'Haenens, D. Kaplan, and P. Merenda, J. Phys. C 8, 2267 (1975); F. Chudnovskii and G. Stefanovich, J. Solid State Chem. 98, 137 (1992).

[15] S. K. Misra, S. I. Andronenko, and R. Andronenko, Phys. Rev. B 57, 8203 (1998).

[16] S. Biermann, A. Poteryaev, A. I. Lichtenstein, and A. Georges, Phys. Rev. Lett. 94, 026404 (2005).

[17] M. Gatti, F. Bruneval, V. Olevano, and L. Reining, Phys. Rev. Lett. 99, 266402 (2007).

[18] C. Weber, D. D. O'Regan, N. D. M. Hine, M. C. Payne, G. Kotliar, and P. B. Littlewood, Phys. Rev. Lett. 108, 256402 (2012).

[19] H. Zheng and L. K. Wagner, Phys. Rev. Lett. 114, 176401 (2015).
[20] V. Eyert, Phys. Rev. Lett. 107, 016401 (2011).

[21] T. J. Huffman, C. Hendriks, E. J. Walter, J. Yoon, H. Ju, R. Smith, G. L. Carr, H. Krakauer, and M. M. Qazilbash, Phys. Rev. B 95, 075125 (2017).

[22] S. Hu, S. Li, R. Ahuja, C. G. Granqvist, K. Hermansson, G. A. Niklasson, and R. H. Scheicher, Appl. Phys. Lett. 101, 201902 (2012).

[23] S. Li, N. R. Mlyuka, D. Primetzhofer, A. Hallén, G. Possnert, G. A. Niklasson, and C. G. Granqvist, Appl. Phys. Lett. 103, 161907 (2013).

[24] A. Krammer, A. Magrez, W. A. Vitale, P. Mocny, P. Jeanneret, E. Guibert, H. J. Whitlow, A. M. Ionescu, and A. Schüler, J. Appl. Phys. 122, 045304 (2017).

[25] F. Iori, M. Gatti, and A. Rubio, Phys. Rev. B. 85, 115129 (2012).

[26] J. Heyd, G. E. Scuseria, and M. Ernzerhof, J. Chem. Phys. 118, 8207 (2003).

[27] L. A. Ladd and W. Paul, Solid State Commun. 7, 425 (1969).

[28] T. Koethe, Z. Hu, M. Haverkort, C. Schlusser, F. Venturini, N. B. Brookes, O. Tjernberg, W. Reichelt, H. H. Hsieh, H. J. Lin, C. Chen, and L. Tjeng, Phys. Rev. Lett. 97, 116402 (2006).

[29] S. Lee, T. L. Meyer, C. Sohn, D. Lee, J. Nichols, D. Lee, S. S. A. Seo, J. W. Freeland, T. W. Noh, and H. N. Lee, APL Mater. 3, 126109 (2015).

[30] R. Grau-Crespo, H. Wang, and U. Schwingenschlögl, Phys. Rev. B 86, 081101(R) (2012).

[31] C. Franchini, J. Phys.: Condens. Matter 26, 253202 (2014).

[32] S. Xu, X. Shen, K. A. Hallman, R. F. Haglund, and S. T. Pantelides, Phys. Rev. B 95, 125105 (2017).

[33] J. Varignon, M. Bibes, and A. Zunger, Nat. Commun. 10, 1658 (2019).

[34] A. Cavalleri, T. Dekorsy, H. H. W. Chong, J. C. Kieffer, and R. W. Schoenlein, Phys. Rev. B 70, 161102(R) (2004).

[35] S. Wall et al., Science 362, 572 (2018).

[36] S. J. Clark, M. D. Segall, C. J. Pickard, P. J. Hasnip, M. J. Probert, K. Refson, and M. C. Payne, Z. Kristallogr. 220, 567 (2005).

[37] Q. Ren, Y. Cai, and Y. Gao, Comput. Mater. Sci. 150, 337 (2013).

[38] A. Jain et al., APL Mater. 1, 011002 (2013).

[39] J. G. Ramirez, T. Saerbeck, S. Wang, J. Trastoy, M. Malnou, J. Lesueur, J.-P. Crocombette, J. E. Villegas, and I. K. Schuller, Phys. Rev. B 91, 205123 (2015). 